\title{
Productos a base de jugo de manzana: aprovechamiento agroindustrial de un excedente
}

\author{
Betancurt, P. (1), Gioscia, D. (1), Bentancor, J. (2), Arcia, P. (2) \\ Contacto: dgioscia@latu.org.uy \\ (1) Departamento de Proyectos Agroalimentarios, Laboratorio Tecnológico del Uruguay (LATU) - (2) Departamento de \\ Bebidas Fermentadas. Laboratorio Tecnológico del Uruguay (LATU)
}

Recibido: 23/6/2008 - Aprobado: 10/10/2008

\begin{abstract}
$\underline{\text { Resumen }}$
Este trabajo aborda una propuesta tecnológica para encontrar salidas de aprovechamiento agroindustrial a los crecientes excedentes de manzanas que presenta el sector de la fruticultura en Uruguay. Se pretende contribuir a la identificación de alternativas de industrialización mediante el desarrollo de una gama de productos a base de manzana que se destinen tanto para consumo en el mercado interno como el de exportación. La variedad de manzana utilizada es la Malus domestica (nombre comercial Cripp's Pink). Dentro de los productos ensayados se encuentran bebidas fermentadas y no fermentables. Los mismos fueron: jugo de manzana gasificado, sidra seca, sidra gasificada diluida, sidra gasificada sin diluir, sidra obtenida por fermentación en botella (Método Champenoise), aguardiente de manzana y jugo de manzana apagado con alcohol etílico. El proceso de elaboración seguido en todos los casos es similar en las etapas primarias, con particularidades específicas en las siguientes de acuerdo al tipo de producto que se trate. Todos los productos obtenidos fueron analizados desde el punto de vista fisicoquímico y sensorial. A través de los resultados obtenidos, la propuesta muestra que desde el punto de vista tecnológico es factible alcanzar productos de calidad y que existe una posibilidad concreta de industrialización. Se espera que este trabajo constituya un principio de diferentes salidas alternativas a un problema importante que se plantea a nivel de la fruticultura, y que sea a su vez detonador de un intercambio constructivo entre todos los interesados, actores de la cadena productiva.

Palabras clave: aprovechamiento agroindustrial, excedentes de manzana, bebidas, industrialización, cadena productiva
\end{abstract}

\begin{abstract}
This is a technological proposal for finding ways to maximize the use of the increasing surplus of apples in the Uruguayan fruit growing industry. This paper is meant as a contribution to identify industrial alternatives, by developing a number of apple based products that may be used for internal consumption and also for export. The variety of apple being used is Malus domestica (its commercial name is Cripp`s Pink). Among the products on trial there were fermented and not fermented beverages, such as: apple juice with soda, dry cider, diluted carbonated cider, carbonated cider with no dilution, bottle fermented cider (Champenoise Method), apple liquor, and apple juice with a touch of ethyl alcohol. In all cases the elaboration process in the primary stages is similar, and in the following stages it gets more specific depending on the product being made. All the products were analyzed from a physicochemical and sensorial point of view. The results obtained have proved that it is technically possible to obtain very good products, and that there is a real possibility for industrialization. We expect to have shown different alternatives to solve an important problem in the fruit growing area and that we hope this may serve as a detonator for a constructive exchange among all the actors in the production area.
\end{abstract}

Key words: agro industrial maximization, apple surplus, beverages, industrialization, productive chain.

\section{Introducción}

En Uruguay se viene observando en los últimos años un incremento de la producción de manzanas que responde al aumento de las plantaciones. En 2005 se realizaron las últimas plantaciones de frutales de hoja caduca en el marco del Programa de Reconversión y Desarrollo de la Granja (PREDEG), lo que explica el alto nivel de inversión del año, ya que los productores hicieron uso de los últimos subsidios del programa.

Como resultado de las plantaciones realizadas en los años recientes, en 2005 se tuvo la máxima producción de manzanas. Ese año el mercado interno debió absorber aproximadamente 67.000 toneladas de fruta, de las cuales 53.000 se destinaron al consumo como fruta fresca y 14.000 a la industrialización (sidra, dulces, pulpas, etc.). Las exportaciones fueron récord histórico, alcanzando un volumen de 11.000 toneladas por un valor de casi 5 millones de dólares. Este nivel de exportaciones representó un aumento de un $30 \%$ en volumen y de un $47 \%$ en términos de valor con respecto al año anterior. Si bien las exportaciones crecen año a año, el incremento no acompasa la mayor oferta de fruta, destinándose para el mercado interno un volumen superior a los niveles de demanda, lo que determina bajos precios del mercado y dificultades de colocación.

El país ya ha superado los 3 millones de plantas, de los cuales un $25 \%$ aún no está en producción y, según las últimas encuestas frutícolas, en este último invierno se plantaron unas 280.000 plantas más, aproximadamente el $9 \%$ del total. En consecuencia, en el mediano plazo la producción seguirá creciendo. Algunas estimaciones realizadas indican que las inversiones en el marco de la reconversión agregarán a la oferta interna en el corto plazo más de 40.000 toneladas de manzana, por lo que es posible esperar que en 2010 la producción nacional supere las 100.000 toneladas anuales.

El consumo interno de fruta fresca absorbe un máximo de 50.000 toneladas para el consumo en fresco y aproximadamente 10.000 para industrializar. De cumplirse las proyecciones de crecimiento de la oferta, será imprescindible colocar en mercados externos entre 40.000 y 50.000 toneladas de fruta. En caso de no poder desarrollarse una estrategia sustentable en el mediano plazo, el rubro puede afrontar serios problemas (Tambler, 2005). Es por ello que se busca destinar estos excedentes de manzana para la elaboración de distintos productos 
que sean comercializables tanto en el mercado interno como externo.

La cosecha de manzana de 2006 fue más baja de lo esperado y de la obtenida el año anterior. La menor producción determinó una caída en las exportaciones, una colocación fluida en el mercado interno, subas notorias de precios hacia el final de la zafra y algunas importaciones para abastecer el mercado en los últimos meses del año. El $79 \%$ de la oferta de manzana se destinó hacia el consumo en fresco (unas 48.000 toneladas), la industria procesó el $9 \%$ (5.000 toneladas) y la exportación un $13 \%$ (7.500 toneladas). El consumo en fresco fue inferior al del año anterior $(-10 \%)$, mientras que el destino industrial cayó 60 \% y la exportación $34 \%$. En general, la industria es un destino de excedentes y de precios muy poco remunerativos, por lo que es el último destino buscado por los productores (Tambler, 2006).

Dados los antecedentes mencionados de aumento en los excedentes de manzana, se espera que este trabajo constituya un principio de diferentes salidas alternativas a este problema que se plantea a nivel de la fruticultura. Se espera también que sea detonador de un intercambio constructivo entre todos los interesados y que genere a partir de los conocimientos y experiencias adquiridas nuevas líneas de acción futura que consoliden respuestas efectivas.

El objetivo del trabajo es, por tanto, contribuir a la identificación de alternativas de industrialización de los descartes de manzana de exportación. Una de las opciones que se plantea es el desarrollo de diferentes productos bebibles fermentados y no fermentables.

Los productos ensayados son:
1. Jugo de manzana clarificado gasificado
2. Sidra seca
3. Sidra gasificada diluida
4. Sidra gasificada sin diluir
5. Sidra obtenida por fermentación en botella
(Método Champenoise)
6. Aguardiente de manzana
7. Jugo de manzana apagado con alcohol etílico

Para el producto a ensayar jugo de manzana gasificado, se toman como base trabajos anteriores desarrollados en el Laboratorio Tecnológico del Uruguay (LATU) (Moyano et al. 1981) y además se toman como referencias otras publicaciones (Ashurst, 1999; Ashurst, 2005) Para el resto de los productos a ensayar no se dispone de antecedentes particulares, por lo cual se hace uso de experiencias adquiridas por parte de los técnicos asignados a este trabajo, además de recurrir a algunas referencias bibliográficas (Rose, 1977; Salas Bringas, 1999; Algood et al.).

\section{Materiales y Métodos}

A continuación se detalla el equipamiento utilizado:

- Balanza de planta, máx. 500 kg, marca FLORENZ

- Lavador de cilindro rotatorio por aspersión, marca LOPEZ CARRILLO

- Tinas de lavado, de acero inoxidable

- Molino de martillo de acero inoxidable, marca AMOS

- Prensa de pistón hidráulico, marca AMOS

- Filtro de placas, marca SCHENK

- Pasteurizador de placas, marca SCHMIDT

- Enfriador tubular, marca Meitar

- Paila de acero inoxidable, de doble camisa, marca Hwolff

- Gasificadora manual de 6 cuerpos

- Tapadora manual

- Colocadora de bozales

- Cámara de refrigeración $\left(5^{\circ} \mathrm{C}\right)$ marca Tupiniquim

- Baño termostatizado, con rango de temperatura desde $-20{ }^{\circ} \mathrm{C}$ a temperatura ambiente

- pHímetro marca Digitalmeter Digi 610 WTW
- Refracómetro marca Abbe Mark II

Los métodos analíticos utilizados son los siguientes:

- pH: basado en método ISO 1842:1991(E).

- ${ }^{\circ}$ Brix: basado en método ISO 2173:2003 (E).

- Acidez total: basado en método ISO/F DIS 750:1998(E).

- Acidez volátil: basado en Compendium of International Methods of Analisys (OIV A11).

- Grado Alcóholico: basado en Compendium of International Methods of Analisys (OIV A2)?

- Azúcares reductores (Fehling): basado en método Cousse Bonnans

- Extracto seco por cálculo densimétrico.

- Anhídrido Sulfuroso libre: basado en Compendium of International Methods of Analysis (OIV A17).

- Anhídrido Sulfuroso total: basado en Compendium of International Methods of Analysis (OIV A17).

- Densidad: basado en Compendium of International Methods of Analysis (OIV A2).

- Análisis Sensorial Escala hedónica estructurada de 9 puntos.

\section{Parte experimental}

La materia prima utilizada, variedad Malus domestica (nombre comercial Cripp's Pink), resultante de excedente de exportación, aportada por un productor de manzanas, fue acondicionada y transportada en bins hacia el LATU donde se la recibe y acondiciona para el tratamiento posterior. Todos los ensayos se llevaron a cabo en la Planta Piloto del LATU, la cual dispone de equipos apropiados para desarrollar a escala experimental las pruebas programadas.

Las etapas primarias de elaboración son similares en todos los casos, pues el insumo inicial es la pulpa de manzana a partir del cual derivan los distintos productos.

\section{Procedimientos}

\section{- Etapas preliminares para la elaboración de la pulpa de manzana base}

Los pasos seguidos para la realización de los ensayos son la recepción, la selección y el pesado.

La manzana se pesa en balanza de planta y se selecciona en función de su estado sanitario, descartándose aquella en avanzado estado de descomposición.

\section{Lavado:}

La materia prima seleccionada recibe un triple tratamiento:

1. Lavado con agua de línea con lavador de cilindro rotatorio por aspersión.

2. Desinfección con agua clorada $50 \mathrm{mg} / \mathrm{L}$ en inmersión en tinas de acero inoxidable.

3. Enjuagado con agua clorada $2 \mathrm{mg} / \mathrm{L}$ por lluvia en lavador cilindro rotatorio por aspersión.

\section{Molienda:}

Se tritura la manzana mediante molino de martillo, recogiéndose la pulpa molida en tarrinas. La pulpa molida obtenida constituye el insumo principal del proceso que caracteriza a cada producto formulado. 


\section{Productos ensayados}

\section{1) Jugo de manzana clarificado gasificado}

Luego de la molienda, prosigue el prensado, el pesado y la colocación en tarrinas.

\section{Prensado:}

La manzana molida se coloca en una prensa de pistón hidráulico de marcos y telas. El jugo comienza a extraerse y se recoge en una bandeja inferior para su posterior recolección en tarrinas.

\section{Clarificación:}

Al jugo obtenido envasado en tarrinas se le adiciona metabisulfito de sodio $(80 \mathrm{mg} / \mathrm{L})$ y bentonita $(1,5 \mathrm{~g} / \mathrm{L})$, con el propósito de reducir la oxidación enzimática y facilitar la clarificación por decantación de la pulpa suspendida durante la fase de reposo.

El bagazo (cáscara, semillas y parte de pulpa retenida en las telas) es pesado y posteriormente descartado. El rendimiento de jugo obtenido en las condiciones de trabajo descritas fue de $66,7 \%$.

\section{Reposo:}

A estos efectos, las tarrinas son colocadas en cámaras de refrigeración entre $2-5^{\circ} \mathrm{C}$, durante $10-12$ horas para favorecer la clarificación del jugo.

\section{Filtrado, pasteurizado y enfriado:}

Una vez clarificado, el jugo es trasegado y filtrado a través de filtro de placas (porosidad $10 \mu \mathrm{m})$. Se agrega sorbato de potasio $(400 \mathrm{mg} / \mathrm{L})$ como conservador químico antimicrobiano, ya que no se dispone de sistema de tratamiento térmico con llenado aséptico. Al jugo se le realiza un tratamiento térmico - pasteurizado-durante tres minutos a $92{ }^{\circ} \mathrm{C}$ en un pasteurizador de placas. El jugo pasteurizado es enfriado en un enfriador tubular hasta alcanzar temperaturas de $4{ }^{\circ} \mathrm{C}$.

\section{Llenado en botella, gasificado, tapado y almacenado:}

El jugo refrigerado se envasa en botellas de vidrio de $750 \mathrm{ml}$, se gasifica mediante gasificadora manual, de tal forma de obtener entre 3 y 4 atmósferas de presión, se tapa utilizando una tapadora manual y se almacena. Los resultados analíticos se presentan en Tablas 1 y 2 (Resultados y Discusión).

\section{2) Sidra seca}

Luego de la molienda, se prosigue con las siguientes etapas:

\section{Prensado, pesado y colocación en tarrinas:}

Se repiten las operaciones anteriores excepto la clarificación. Esta vez al jugo obtenido mantenido en tarrinas se le adiciona metabisulfito de sodio $(80 \mathrm{mg} / \mathrm{L})$, enzima pectolítica derivada de Aspergillus níger ( $4 \mathrm{mg} / \mathrm{L}$ ) y levadura Saccharomyces cerevisiae $(200 \mathrm{mg} / \mathrm{L})$.

El bagazo es pesado y posteriormente descartado. Los rendimientos de jugo son similares al caso anterior y se repiten para los siguientes productos fermentados.

\section{Fermentación:}

A estos efectos, las tarrinas son colocadas en Planta Piloto a temperatura ambiente, donde comienza el proceso de transformación biológico química del azúcar natural del jugo en alcohol. El criterio adoptado en forma práctica y sencilla para decidir la finalización de la fermentación fue el valor de los ${ }^{\circ}$ Brix o \% de Sólidos Solubles (expresados como gramos de sacarosa por $100 \mathrm{~g}$ de producto), partiendo de $14.5^{\circ}$ Brix y llegando a aproximadamente $5{ }^{\circ} \mathrm{Brix}$.

\section{Trasiego, clarificado y filtrado:}

Una vez alcanzados los $5^{\circ}$ Brix, se procede al primer trasiego, para luego incorporar bentonita $(1.5 \mathrm{~g} / \mathrm{L})$ como agente clarificante, proceso que se realiza a temperatura ambiente. Luego de clarificado, se trasiega y agrega metabisulfito de sodio $(100 \mathrm{mg} / \mathrm{L})$, sorbato de potasio $(300 \mathrm{mg} / \mathrm{L})$ y ácido cítrico $(300 \mathrm{mg} / \mathrm{L})$. Por último es filtrado a través de filtro de placas (porosidad $10 \mu \mathrm{m}$ ).

\section{Llenado en botella, tapado y almacenado:}

La sidra se envasa en botellas de vidrio de $750 \mathrm{ml}$, se tapa, mediante tapadora manual y se almacena. Los resultados analíticos se presentan en Tablas 1 y 2 (Resultados y Discusión).

\section{3) Sidra gasificada diluida}

Todo el proceso previo hasta el trasiego es similar al caso anterior (sidra seca), al igual que el rendimiento en jugo obtenido.

\section{Trasiego, clarificado y filtrado:}

Una vez clarificado, el fermentado se trasiega y agrega metabisulfito de sodio $(150 \mathrm{mg} / \mathrm{L})$, sorbato de potasio $(450 \mathrm{mg} / \mathrm{L})$, ácido cítrico (300 $\mathrm{mg} / \mathrm{L})$, agua (0.5 litros de agua/ Litro de sidra seca) y azúcar (100 g de azúcar/Litro de sidra seca). Por último se filtra a través de filtro de placas (porosidad $10 \mu \mathrm{m}$ ).

(Nota: la cantidad de agua agregada fue determinada en base a un cálculo teórico de grado alcohólico).

\section{Llenado en botella, gasificado, tapado y almacenado:}

La sidra se envasa en botellas de vidrio de $750 \mathrm{ml}$ y refrigera en cámara hasta llegar a una temperatura entre $5-8{ }^{\circ} \mathrm{C}$, procediéndose entonces a la gasificación, mediante gasificadora manual, de tal forma de obtener entre 3 y 4 atmósfera de presión. Finalmente se tapa, con tapadora manual y se almacena. Los resultados analíticos se presentan en Tablas 1 y 2 (Resultados y Discusión).

\section{4) Sidra gasificada sin diluir}

El proceso es el mismo que el de obtención de sidra gasificada diluida, hasta el segundo trasiego.

\section{Trasiego y filtrado:}

El fermentado clarificado se trasiega y agrega metabisulfito de sodio $(100 \mathrm{mg} / \mathrm{L})$, sorbato de potasio $(300 \mathrm{mg} / \mathrm{L})$, ácido cítrico $(300$ $\mathrm{mg} / \mathrm{l})$ y azúcar (100 g de azúcar/ Litro de sidra seca). Por último se filtra a través de filtro de placas (porosidad $10 \mu \mathrm{m}$ ).

Llenado en botella, gasificado, tapado y almacenado:

Se procede de igual forma que para sidra gasificada diluida. Los resultados analíticos se presentan en Tablas 1 y 2 (Resultados y Discusión)

\section{5) Sidra fermentación en botella (método Champenoise)}

El proceso se repite hasta el segundo trasiego, cuando el fermentado ha sido clarificado.

\section{Trasiego, filtrado y $2^{\text {a }}$ fermentación:}

Una vez clarificado, el producto fermentado es trasegado y filtrado a través de filtro de placas (porosidad $10 \mu \mathrm{m}$ ). Luego es colocado en tarrinas en donde se le agrega levadura Saccharomyces cerevisiae (300 $\mathrm{mg} / \mathrm{L}$ ) y azúcar (25 g de azúcar/Litro de sidra seca) para dar comienzo a la segunda fermentación. También se le adiciona bentonita $(1.5 \mathrm{~g} / \mathrm{L})$ para favorecer el arrastre de las borras.

\section{Envasado preliminar y reposo:}

El llenado en botella se realiza cuando da comienzo la segunda fermentación. La sidra es envasada en botellas de vidrio de $750 \mathrm{ml}$ y tapada con cápsula contenedora de borras y tapa corona. Las botellas son colocadas en posición horizontal en cajones y conservadas a temperatura ambiente. El criterio empleado para la colocación de botellas en pupitres fue la presencia de gas carbónico producido por la 
fermentación dentro de la botella al destaparla. Las botellas en pupitres se conservan en sala climatizada a $21^{\circ} \mathrm{C}$, rotando los envases una vez al día para favorecer el desplazamiento de las borras hacia la cápsula contenedora ubicada en el pico de la botella.

\section{Degollado, tapado y almacenado:}

Una vez que las borras se ubican en la cápsula contenedora, las botellas se trasladan en sus pupitres a cámara de refrigeración $\left(2-5^{\circ} \mathrm{C}\right)$, para bajar la temperatura del líquido y del envase de forma uniforme, minimizando los tiempos posteriores de congelado del pico. Luego se procede al congelado de los cuellos de las botellas, mediante el uso de baño termostatizado con solución salina al $10 \%$. Dicho baño alcanzó temperaturas de $-20^{\circ} \mathrm{C}$ en la solución. Una vez congelado el pico, se procede al degollado de los mismos para retirar las borras. A cada botella de $750 \mathrm{ml}$ se le agrega $50 \mathrm{ml}$ de licor de expedición *. Luego, de ser necesario, se completa el volumen de cada botella con sidra de la misma partida.

Para este proceso de fabricación se siguen los conceptos originales de la elaboración tradicional de vinos cava por el método Champenoise. El mismo contempla en su gama de productos, la posibilidad de agregar licor de expedición, obteniendo de esta manera la variante demi-sec.

Por tanto, el objetivo de usar un licor de expedición en esta experiencia es obtener un bouquet diferente, usando un producto como el aguardiente de manzana originario de la propia fruta.

Finalmente se tapa y almacena a temperatura ambiente. Los resultados analíticos se presentan en Tablas 1 y 2 (Resultados y Discusión).

* El licor de expedición para cada botella $(50 \mathrm{ml})$ está compuesto de aguardiente de manzana $43^{\circ} \mathrm{G} . \mathrm{L} .(24.6 \mathrm{ml})$, azúcar $(36.9 \mathrm{~g})$ y agua (3.3 ml).

\section{6) Aguardiente de manzana}

El procedimiento para la obtención del jugo y las etapas siguientes hasta la finalización de la fermentación cuando el producto alcanza los $5{ }^{\circ}$ Brix. Luego se procede como sigue:

\section{Trasiego y clarificado:}

Se procede al primer trasiego y luego se incorpora bentonita (1.5 $\mathrm{g} / \mathrm{L})$ como agente clarificante.

\section{2do Trasiego y colocación en tarrinas:}

Una vez clarificado, se trasiega nuevamente y se coloca en tarrinas en donde se le agrega metabisulfito de sodio (2000 ppm) para su conservación, dado que su destino no será el consumo directo. A las borras obtenidas en este proceso se le incorporan las obtenidas en el resto de las experiencias. Se agrega metabisulfito de sodio a razón de 2000 ppm para su conservación. Una vez que las borras vuelven a sedimentar, se obtienen los claros de borras. Estos claros se agregan a la sidra seca descrita en la etapa anterior (trasiego y clarificado), de modo de aumentar el volumen para su posterior utilización.

\section{Destilado:}

El total de la sidra seca obtenida en este proceso es destilada en una paila de acero inoxidable, de doble camisa, modificada a efectos de simular un destilador. Se parte de una sidra seca con $7.8^{\circ} \mathrm{G}$.L., ajustándose los volúmenes para obtener un destilado con $43^{\circ} \mathrm{G}$.L. En las condiciones de la experiencia, son necesarios $5.5 \mathrm{~L}$ de sidra seca para obtener $1 \mathrm{~L}$ de aguardiente de manzana.

Envasado y almacenado:

El destilado es envasado en botellas de vidrio de $500 \mathrm{ml} \mathrm{y}$ almacenado a temperatura ambiente. Los resultados analíticos se presentan en Tablas 1 y 2 (Resultados y Discusión).

\section{7) Jugo de manzana apagado con alcohol etílico}

En este caso particular, el jugo extraído luego del prensado, recibe un procesamiento diferente que consiste en la adición de alcohol etílico $95^{\circ}$ (en relación jugo:alcohol 5:1) y bentonita $(1,5 \mathrm{~g} / \mathrm{L})$. Posteriormente pasa a cámara de refrigeración para reposar.

\section{Reposo en cámara de refrigeración:}

Las tarrinas con el jugo tratado son colocadas en cámaras de refrigeración entre $2-5^{\circ} \mathrm{C}$ para favorecer la clarificación del producto.

\section{Trasiego y filtrado:}

El jugo, una vez clarificado y trasegado, es filtrado a través de filtro de placas (porosidad $10 \mu \mathrm{m}$ ).

Envasado y almacenado:

El jugo se envasa en botellas de vidrio de $750 \mathrm{ml}$ y almacena a temperatura ambiente. Los resultados analíticos se presentan en Tablas 1 y 2 (Resultados y Discusión).

\section{Resultados y Discusión}

Todos los productos obtenidos fueron analizados desde el punto de vista fisicoquímico y sensorial.

\section{Análisis fisicoquímicos}

Los resultados de los análisis fisicoquímicos de todos los productos se presentan en la Tabla 1.

\begin{tabular}{|c|c|}
\hline Análisis & Resultado \\
\hline \multicolumn{2}{|c|}{ Jugo de manzana clarificado gasificado } \\
\hline $\mathrm{pH}$ & 4.20 \\
\hline${ }^{\circ}$ Brix & 13.6 \\
\hline Análisis & Resultado \\
\hline \multicolumn{2}{|c|}{ Sidra seca } \\
\hline $\mathrm{pH}$ & 3.78 \\
\hline${ }^{\circ}$ Brix & 4.91 \\
\hline Acidez volátil & $0.25 \mathrm{~g}$ acet $/ \mathrm{L}$ \\
\hline Acidez total & $3.49 \mathrm{~g} \operatorname{tart} / \mathrm{L}$ \\
\hline Azúcares reductores & $2.3 \mathrm{~g}$ gluc $/ \mathrm{L}$ \\
\hline Extracto seco & $18.7 \mathrm{~g} / \mathrm{L}$ \\
\hline Anhídrido Sulfuroso libre & $6 \mathrm{mg} / \mathrm{L}$ \\
\hline Anhídrido Sulfuroso total & $70 \mathrm{mg} / \mathrm{L}$ \\
\hline Densidad & $0.99615 \mathrm{~g} / \mathrm{ml}$ \\
\hline Grado alcohólico & $7.92 \% \mathrm{vol}$ \\
\hline \multicolumn{2}{|c|}{ Sidra gasificada diluida } \\
\hline $\mathrm{pH}$ & 3.70 \\
\hline${ }^{\circ}$ Brix & 12.77 \\
\hline Acidez volátil & $0.25 \mathrm{~g}$ acet $/ \mathrm{L}$ \\
\hline Acidez total & $2.64 \mathrm{~g} \operatorname{tart} / \mathrm{L}$ \\
\hline
\end{tabular}




\begin{tabular}{|c|c|}
\hline Azúcares reductores & $114.2 \mathrm{~g}$ gluc $/ \mathrm{L}$ \\
\hline Extracto seco & $120.1 \mathrm{~g} / \mathrm{L}$ \\
\hline Anhídrido Sulfuroso libre & $8 \mathrm{mg} / \mathrm{L}$ \\
\hline Anhídrido Sulfuroso total & $68 \mathrm{mg} / \mathrm{L}$ \\
\hline Densidad & $1.03834 \mathrm{~g} / \mathrm{mL}$ \\
\hline Grado alcohólico & $4.22 \% \mathrm{vol}$ \\
\hline \multicolumn{2}{|c|}{ Sidra gasificada sin diluir } \\
\hline $\mathrm{pH}$ & 3.77 \\
\hline${ }^{\circ}$ Brix & 14.34 \\
\hline Acidez volátil & $0.27 \mathrm{~g}$ acet $/ \mathrm{L}$ \\
\hline Acidez total & $3.53 \mathrm{~g} \operatorname{tart} / \mathrm{L}$ \\
\hline Azúcares reductores & $114.2 \mathrm{~g}$ gluc/L \\
\hline Extracto seco & $128.2 \mathrm{~g} / \mathrm{L}$ \\
\hline Anhídrido Sulfuroso libre & $7 \mathrm{mg} / \mathrm{L}$ \\
\hline Anhídrido Sulfuroso total & $80 \mathrm{mg} / \mathrm{L}$ \\
\hline Densidad & $1.03714 \mathrm{~g} / \mathrm{ml}$ \\
\hline Grado alcohólico & $7.20 \% \mathrm{vol}$ \\
\hline
\end{tabular}

\begin{tabular}{|c|c|}
\hline \multicolumn{2}{|c|}{ Sidra fermentación en botella } \\
\hline $\mathrm{pH}$ & 3.88 \\
\hline${ }^{\circ}$ Brix & 9.80 \\
\hline Acidez volátil & $0.20 \mathrm{~g}$ acet $/ \mathrm{L}$ \\
\hline Acidez total & $2.75 \mathrm{~g} \operatorname{tart} / \mathrm{L}$ \\
\hline Azúcares reductores & $61.4 \mathrm{~g}$ gluc/L \\
\hline Extracto seco & $71.8 \mathrm{~g} / \mathrm{L}$ \\
\hline Anhídrido Sulfuroso libre & $4 \mathrm{mg} / \mathrm{L}$ \\
\hline Anhídrido Sulfuroso total & $48 \mathrm{mg} / \mathrm{L}$ \\
\hline Densidad & $1.01316 \mathrm{~g} / \mathrm{mL}$ \\
\hline Grado alcohólico & $9.10 \% \mathrm{vol}$ \\
\hline \multicolumn{2}{|c|}{ Aguardiente de manzana } \\
\hline Alcohol metílico & $2,7 \mathrm{ml} / \mathrm{L}$ \\
\hline Grado alcohólico & $42.7 \% \mathrm{vol}$ \\
\hline \multicolumn{2}{|c|}{ Jugo de manzana apagado con alcohol etílico } \\
\hline $\mathrm{pH}$ & 4.07 \\
\hline${ }^{\circ}$ Brix & 17.41 \\
\hline Acidez volátil & $0.02 \mathrm{~g}$ acet $/ \mathrm{L}$ \\
\hline Azúcares reductores & $129.8 \mathrm{~g}$ gluc $/ \mathrm{L}$ \\
\hline Densidad & $1.02677 \mathrm{~g} / \mathrm{ml}$ \\
\hline Grado alcohólico & $16.08 \% \mathrm{vol}$ \\
\hline
\end{tabular}

Tabla 1. Resultados de análisis fisicoquímicos.

\section{Análisis sensorial}

A cada producto se lo somete a una degustación para evaluar su grado de aceptabilidad comercial. La prueba de evaluación sensorial es realizada por un grupo de consumidores. El parámetro evaluado fue el agrado general. El nivel de aceptación se mide utilizando una escala hedónica de nueve puntos del 1 al 9, donde 1 es Me disgusta extremadamente y 9 es Me gusta extremadamente.

Se toma como criterio para que un producto sea aceptable que éste reciba puntajes iguales o mayores a 5 . A continuación se presenta la escala hedónica de 9 puntos utilizada:
1 - Me disgusta extremadamente
2 - Me disgusta mucho
3 - Me disgusta moderadamente
4 - Me disgusta ligeramente
5 - Ni me gusta ni me disgusta
6 - Me gusta ligeramente
7 - Me gusta moderadamente
8 - Me gusta mucho
9 - Me gusta extremadamente

En la Tabla 2 se presentan los puntajes obtenidos para cada producto desarrollado, que corresponden al valor promedio entre todos los juicios.

\begin{tabular}{|c|c|}
\hline Producto & Resultado \\
\hline Jugo de manzana clarificado gasificado & 7.1 \\
\hline Sidra seca & 4.8 \\
\hline Sidra gasificada diluida & 6.9 \\
\hline Sidra gasificada sin diluir & 7.3 \\
\hline Sidra fermentación en botella & 7.1 \\
\hline Aguardiente de manzana & 5.5 \\
\hline Jugo de manzana apagado con alcohol etílico & 5.6 \\
\hline
\end{tabular}

Tabla 2. Análisis sensorial.

\section{1) Jugo de manzana clarificado gasificado}

De acuerdo a los resultados sensoriales, el producto es aceptado por los consumidores. Se podría estimar que cumple con las expectativas planteadas de producto natural levemente ácido, gasificado y sin el agregado de colorantes, saborizantes o azúcares. Podría comercializarse dentro de la gama de refrescos o bebidas refrescantes gasificadas $\sin$ alcohol

Si bien para los ensayos se utilizaron envases de vidrio, el jugo podría comercializarse en envases Pet.

\section{2) Sidra seca}

Según los resultados sensoriales, el producto no es aceptado por los consumidores. Este resultado podría estar relacionado a la inexistencia de productos similares en el mercado interno, por lo que el consumidor no tendría parámetros de comparación.

\section{3) Sidra gasificada diluida}

Si bien los resultados sensoriales son positivos en cuanto al 
grado de aceptabilidad, con respecto a los resultados fisicoquímicos, el producto se acerca a los límites especificados en el Reglamento Bromatológico Nacional en lo que a grado alcohólico refiere (límite de $4,5 \%$ vol de alcohol), impidiendo su comercialización.

\section{4) Sidra gasificada sin diluir}

De acuerdo a los resultados sensoriales, esta sidra es aceptada por los consumidores, obteniendo el mayor puntaje entre todos los productos ensayados. Si se cotejan los resultados entre sidra gasificada sin diluir y la diluida (Tabla 1), se observa que las diferencias en acidez y grado alcohólico son menores para la sidra diluida, sin presentar diferencias en azúcares reductores.

Una de las conclusiones que podemos derivar es que el consumidor perciba y prefiera una sidra con mayor grado alcohólico y sus demás componentes naturalmente balanceados.

\section{5) Sidra fermentación en botella}

Según los resultados sensoriales, el producto obtenido es aceptado por los consumidores, sin embargo se deben hacer las siguientes precisiones:

- El momento del año disponible para la ejecución de este ensayo (fin del invierno) evitó la correcta fermentación secundaria a temperatura ambiente.

- El producto final presenta turbidez. La misma se debe a presencia de borra, consecuencia de dos factores:

1) el tiempo en pupitre empleado para la precipitación de las borras hacia el cuello no fue suficiente.

2) el uso de muy bajas temperaturas en el congelado del cuello previo al degollado causó que las borras se pegaran al vidrio del mismo, evitando su correcto desplazamiento hacia el exterior.

Por lo dicho, el proceso y metodología empleados para la obtención de este producto pueden ser mejorados.

\section{6) Aguardiente de manzana}

Según los resultados sensoriales, este producto es aceptado levemente por los consumidores. Analizando los puntajes asignados por los evaluadores, se observa gran variabilidad entre los mismos, donde un $40.7 \%$ opinó que le gusta ligeramente. Los valores de alcohol metílico registrados están por encima de lo permitido por la reglamentación (valor máximo permitido $2,0 \mathrm{ml} / \mathrm{L}$ ).

\section{7) Jugo de manzana apagado con alcohol etílico}

Esta es una bebida a base de jugo de manzana a la que no se le permite fermentar mediante el agregado de alcohol etílico, alcanzando valores entre 16-19\%vol. Esta característica le da perfil de licor para postres o aperitivos.

Según los resultados sensoriales, el producto obtenido es aceptado levemente por los consumidores. Al analizar los puntajes asignados por los evaluadores, se observa gran variabilidad entre los mismos, donde un $66 \%$ emitió juicios con puntaje iguales o mayores a 6 .

Dentro de los juicios de los consumidores se leen comentarios como: "algo fuerte de más por el alcohol", "me resultó con mucho gusto a alcohol", "demasiado alto el porcentaje alcohólico", "no se distingue el gusto a manzana, mucho gusto a alcohol". Esto puede deberse a que el producto no se dejó reposar lo suficiente como para que se encuentren balanceados los componentes y a la clara falta de acidez en el jugo de manzana. Cabe aclarar que para el caso de la Mistela (jugo de uva apagado con alcohol etílico), es usual un reposo de 6 meses a partir de la fecha de elaboración.

A continuación se presentan algunas recomendaciones sobre aspectos particulares de cada producto, que deberían tenerse en cuenta en futuros desarrollos para mejorar su aceptabilidad y adecuarlos en los casos que correspondiera a la normativa vigente.

\section{1) Jugo de manzana gasificado}

Si bien los valores de sólidos solubles y $\mathrm{pH}$ son adecuados para este tipo de producto, se recomienda reducir el $\mathrm{pH}$ acidificando el medio para llegar a valores en el entorno de 3.9-4.0 a fin de aumentar el rango de seguridad del producto. Se considera que este cambio haría más atractivo el producto desde el punto de vista sensorial.

\section{2) Sidra seca}

Para lograr cotejar los resultados sensoriales, se recomienda en futuras experiencias conseguir muestras de este tipo de producto en países donde el mismo se comercialice.

\section{3) Sidra gasificada diluida}

La recomendación para futuras experiencias es ajustar el grado alcohólico (a valores reglamentarios), acidez y azúcar (sacarosa agregada).

\section{4) Sidra gasificada sin diluir}

En vista de los resultados obtenidos a nivel sensorial, se recomienda que los futuros ensayos incluyan otras variedades de manzana para obtener sidras varietales o cortes en función de los volúmenes existentes.

\section{5) Sidra fermentación en botella}

Para futuras experiencias se recomienda:

- Iniciar la fermentación secundaria inmediatamente después de la primaria, entre marzo y abril.

- Respetar los tiempos en pupitres dispuestos para la elaboración de vinos cava por el método Champenoise.

- Ajustar la temperatura del baño de inmersión donde se realiza el congelado de las borras de los cuellos de las botellas, para que las mismas se retiren fácilmente.

Es recomendable el agregado de sorbato de potasio en el licor de expedición como agente conservador.

\section{6) Aguardiente de manzana}

Debido a la variabilidad en los puntajes asignados por los evaluadores sensoriales, se recomienda recurrir a consumidores habituales de este tipo de bebidas. Para aumentar los rendimientos (litros aguardiente/litros sidra seca), se sugiere el estudio de variedades de levaduras que cumplan con este requisito.

\section{7) Jugo de manzana apagado con alcohol etílico}

En este caso se deben realizar estudios que determinen el tiempo de reposo necesario previo al envasado.

Otro factor a considerar es la variedad de manzana. Se podría ensayar con variedades comerciales más aromáticas, por ejemplo Red Delicius, o cortes de ésta con Granny Smith (por su acidez). También es interesante el estudio de correcciones de acidez mediante agregado de ácido málico, ácido cítrico o de ambos ácidos a la vez, teniendo en cuenta parámetros fisicoquímicos y sensoriales.

Otra variable a evaluar es el uso de otro tipo de alcohol, como por ejemplo alcohol de cereales, alcoholes desodorizados a través de prácticas como el uso de carbón o el uso de alcoholes obtenidos por la destilación de sidra seca (aguardiente de manzana), con el objetivo de disminuir el fuerte "aroma a alcohol" descrito por algunos evaluadores sensoriales. 


\section{Conclusiones}

Los productos obtenidos y evaluados tanto analítica como sensorialmente sugieren una posibilidad concreta de industrialización.

En líneas generales se estima que los resultados obtenidos permiten acercar una probable alternativa de aprovechamiento comercial de los excedentes de manzana de exportación, factible, a su vez, desde el punto de vista tecnológico.

La decisión final acerca de su pertinencia productiva e industrial será resultado de una evaluación más pormenorizada de su factibilidad económica y comercial, desarrollada por los grupos interesados y afectados por la situación: productores de manzana y elaboradores de sidra.

\section{Referencias}

- ALGOOD, Tammy ; MORRIS, William; LOCKWOOD, David. Fruit and nuts: home apple cider production [En línea]. University of Tennesee: Tennesee, [s.d.]. [Consulta 24 de setiembre de 2008]. Disponible en: http://www.utextension.utk.edu/publications/spfiles/ SP307-O.pdf

- ASHURST, P. R. Chemistry and technology of soft drinks and fruit juices. 2a. ed. Oxford: Blackwel, 2005.

- ASHURST, P. R. Production and packaging of noncarbonated fruit juices and fruit beverages. $2 \mathrm{a}$ ed. Maryland: Aspen, 1999.

- MOYANO, C., et al. Jugo natural integral de manzana. Montevideo: Laboratorio Tecnológico del Uruguay, 1981. (Monografías Tecnológicas. Serie Frutas y Hortalizas; 2)

- ROSE, A. H. Alcoholic beverages. New York: Academic Press, 1977. (Economic microbiology ; 1)

- SALAS BRINGAS, Mónica. Procesamiento y calidad de sidra en Valdivia-Chile [En línea]. En: Agro Sur. 1999, 27(1): 1-9. [Consulta: 06 de octubre de 2008]. Disponible en: http://mingaonline.uach.cl/scielo.php?script=sci arttext\&pid=S0304-88021999000100001\&lng=es\&nrm=iso

- TAMBLER, A. Frutales de hoja caduca: situación actual y perspectivas [En línea]. En: Anuario 2005. Montevideo: Ministerio de Ganadería Agricultura y Pesca. OPYPA, 2005. [Consulta: 24 de setiembre de 2008]. Disponible en: http://www.mgap.gub.uy/opypa/ANUARIOS/Anuario05/ CadenasProductivas/frutales $\% 20$ hoja $\% 20$ cadu.pdf

- TAMBLER, A. Frutales de hoja caduca: situación actual y perspectivas [En línea]. En: Anuario 2006. Montevideo: Ministerio de Ganadería Agricultura y Pesca. OPYPA. 2006. [Consulta: 24 de setiembre de 2008]. Disponible en: http://www.mgap.gub.uy/opypa/ANUARIOS/Anuario06/ docs/19\%20-\%20FRUTALES\%20T.pdf 